\title{
In-situ NiO nanostructure growth during heating in water vapor atmosphere
}

Boyi Qu and Klaus van Benthem

University of California, Davis, United States

Oxidation behavior of bulk nickel in water vapor atmosphere was previously documented with the formation of different surface features including whiskers and pits [1]. However, transition metal nanoparticles possess unique physical and chemical properties compared to bulk due to their high surfaceto-volume ratio. Anisotropic growth of $\mathrm{NiO}$ from nickel nanoparticles due to rapid thermal annealing in oxygen was reported [2]. The growth of $\mathrm{NiO}$ exhibits either nanocube or nanorod morphologies depending on the oxidation condition and particle size [2].

In this study, in-situ heating experiments were carried out for nickel nanoparticles with a nominal diameter of $300 \mathrm{~nm}$ using a Thermofisher Quattro Environmental Scanning Electron Microscope (ESEM). Isothermal heating experiments were performed at $800{ }^{\circ} \mathrm{C}$ in atmospheres of dry nitrogen or water vapor pressures ranging between 250 and $400 \mathrm{~Pa}$. A Bruker Quantax 400 XFlash 6 solid-state detector was used to acquire Energy dispersive x-ray spectroscopy (EDXS) data before and after in-situ heating. Figure 1(a) and (c) show the as-received nickel particles acquired at room temperature before ESEM heating. The particles show round geometry and smooth surfaces. EDXS data demonstrates a nickel to oxygen atomic concentration ratio of around 95:5, which indicates a native nickel oxide layer present on the nickel particle surface $[3,4]$.

Figure 1(b) and 1(d) were recorded at $800{ }^{\circ} \mathrm{C}$ during heating. Both particle elongation (Figure $1 \mathrm{~b}$ ) and high aspect ratio nanorod growth (Figure 1d) was observed. Spectroscopy and SEM imaging confirmed growth of stoichiometric $\mathrm{NiO}$ with rates below $1.8 \mathrm{~nm} / \mathrm{s}$.

Nanostructure growth is facilitated by the adsorption of oxidant molecules from the gas phase in conjunction with outward nickel diffusion towards the particle surface. High aspect ratio nanorods form where grain boundaries as fast diffusion pathways through the polycrystalline native surface oxide layer are available. Vacuum annealing of nanoparticles prior to in-situ heating experiments revealed that nanostructure growth can only occur on particle surfaces with sufficiently high surface energies so that the addition of nanostructured $\mathrm{NiO}$ can effectively lower total surface energies.

\section{Acknowledgement:}

This work was supported by the Army Research Office under award W911-NF-1610364 (program manager: Dr. Michael Bakas) and the National Science Foundation under award DMR-1836571. The ESEM instrument was acquired with NSF-MRI award DMR-1725618. 

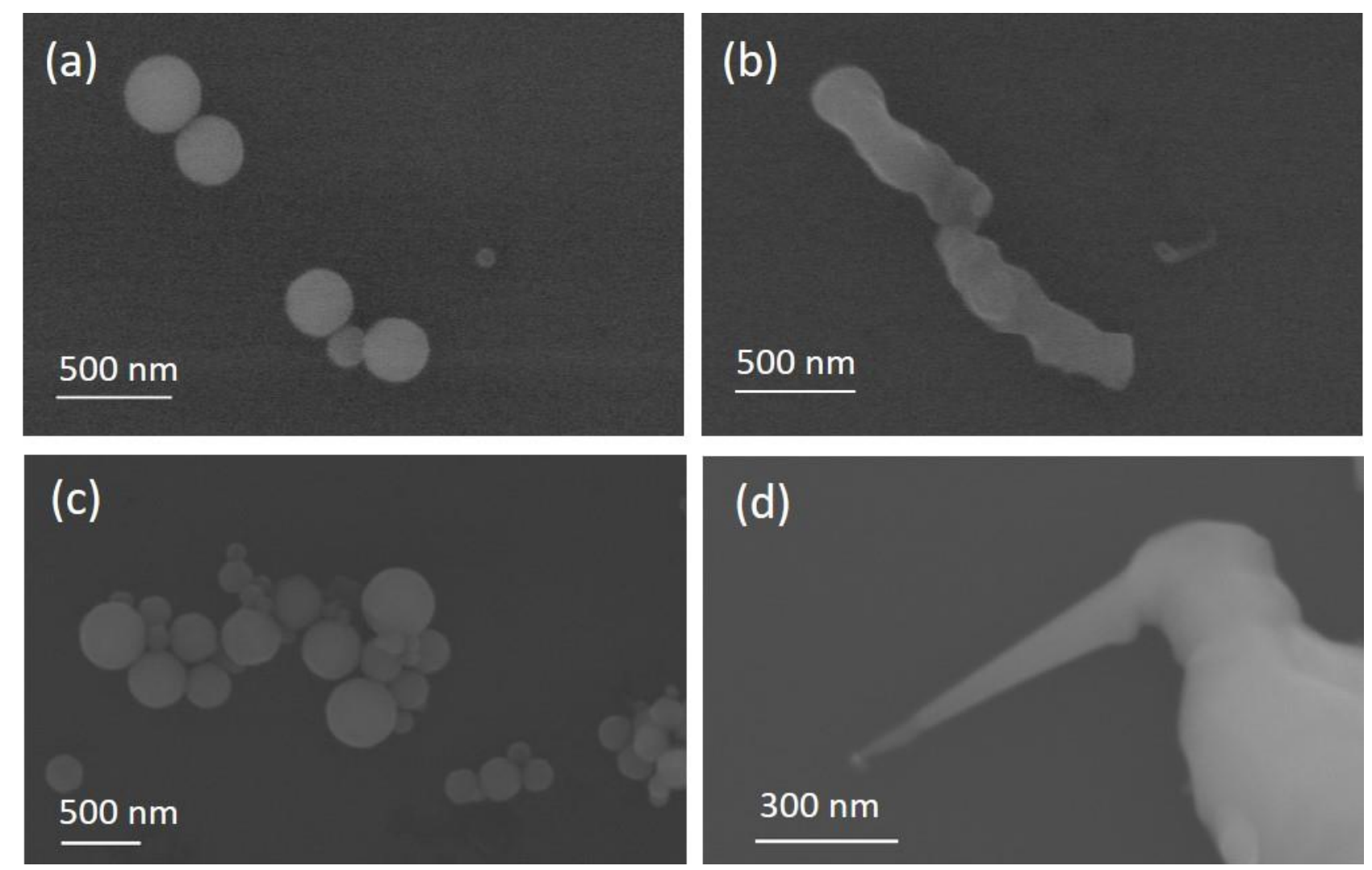

Figure 1. ESEM images of nickel nanoparticles taken at room temperature (a) and (c) and at $800{ }^{\circ} \mathrm{C}(\mathrm{b})$ and (d) during in-situ heating.

\section{References}

[1] G.M. Raynaud, R.A. Rapp, In situ observation of whiskers, pyramids and pits during the hightemperature oxidation of metals, Oxid. Met. 21 (1984) 89-102. https://doi.org/10.1007/BF00659470.

[2] K. Koga, M. Hirasawa, Anisotropic growth of $\mathrm{NiO}$ nanorods from Ni nanoparticles by rapid thermal oxidation, Nanotechnology. 24 (2013) 375602. https://doi.org/10.1088/0957-4484/24/37/375602. [3] M. Matsuno, C.S. Bonifacio, J.F. Rufner, A.M. Thron, T.B. Holland, A.K. Mukherjee, K. van Benthem, In situ transmission electron microscopic investigations of reduction-oxidation reactions during densification of nickel nanoparticles, J. Mater. Res. 27 (2012) 2431-2440. https://doi.org/10.1557/jmr.2012.256.

[4] J.G. Railsback, A.C. Johnston-Peck, J. Wang, J.B. Tracy, Size-Dependent Nanoscale Kirkendall Effect During the Oxidation of Nickel Nanoparticles, ACS Nano. 4 (2010) 1913-1920. https://doi.org/10.1021/nn901736y. 